\title{
TELEMEDICINE PLATFORMS AS A COMPLEX SYSTEM: MODEL AND PROTOTYPING APPROACH
}

\author{
O. S. MORALES, G. TARAZONA \& CÉSAR A. PERDOMO CH \\ Engineering Faculty, Universidad Distrital Francisco José de Caldas, Bogotá, Colombia
}

\section{ABSTRACT}

This document describes in detail the modeling, design and testing of a prototype for the validation of a frame of reference in the development of telemonitoring platforms. In the first section, an introduction is made to the complex system that is desired to model and the needs that gave rise to this work are stated. In the second section, the methodology and the steps followed to develop the prototype are described. Section three shows the results obtained from the tests performed on the prototype developed in Python locally and on the Amazon Web Services server (AWS). Then in section four, a comparison is made with some reference works found in the literature and finally, in section five the conclusions of the work carried out are described.

KEYWORDS: AWS, Biomedical Signals, IoT, Monitoring, Processing, Python, Signal, Telemedicine

Received: Aug 28, 2020; Accepted: Sep 18, 2020; Published: Nov 12, 2020; Paper Id.: IJMPERDOCT202051

\section{INTRODUCTION}

The level of overcrowding of hospitals and health care centers in Colombia and especially in the cities far away from the main capitals has had an increase in recent years, which generates a constant and growing discomfort in the Colombian population due to the delayed or lack of provision of various medical services and access difficulties, especially in remote areas.

The demographic picture is not encouraging, according to the statistics provided by the National Administrative Department of Statistics (DANE) of the last census conducted in 2018 the Colombian population is aging, $9.23 \%$ of the population is over 60 years old [1]. The population of 60 years or more has growth rates higher than the growth of the total population, which means that the aging rate defined as the weight of the adult population regarding the child and adolescent population tripled in the last decades. As highlighted in [2], the goal is to prevent complications and help the elderly maintain their independence in daily life activities through the use of technology, otherwise taking care of the elderly will require more expenditure and time of the economically active population.

At the time of the development of this document, different approaches to reference frames for smart cities can be found in the bibliography, which describes in a general and high-level way the parameters and essential components to implement the new technologies and tendency of the 4th industrial revolution for the benefit and improvement in the quality of life of citizens. But there is no detailed guide for the implementation of technological platforms focused on telemonitoring operating in the cloud, therefore this is one of the main motivations to develop this work.

In this document, we propose a process model developed through the Python programming language. The 
methodological steps for the design and implementation of a scalable telemedicine platform with dynamic data processing capabilities are detailed.

\section{MATERIALS AND METHODS}

To carry out the design and development of the model of the telemonitoring platform of biomedical signals in real-time proposed in this document, five methodological phases were followed. The first step is to make an abstraction of reality to model the complex system that we want to solve, then generate a prototype using this model and finally perform a validation of this prototype with unit tests and performance tests in the cloud through a virtual server. The five phases are listed below:

- Data source: three data sources were used, the databases of the Ministry of Health of Colombia MINSALUD, the databases of Physionet and the repository of machine learning of the University of California, School of Information and Science of Computing.

- System modeling: five different types of models were developed to simulate the complex system.

- Design of a prototype: one of the models designed in the previous phase was selected and a prototype was designed using the Python programming language. This software was chosen for its power, scalability and easy way of programming.

- Prototype tests: through the databases, the correct operation of the prototype was proved, and the first results of the local processing were obtained.

- Assembly of the system in the cloud: using the powerful infrastructure of Amazon Web Services (AWS), a server machine was created, the prototype programs were loaded, and the correct functioning of the system was validated processing in the cloud.

\subsection{Modeling the Complex System}

To make an abstraction of reality by contemplating the most relevant elements of the complex system that we want to model, five models are proposed: analogical, conceptual, graphic, statistical and processes. This last model was used as the basis to build the prototype in the Python programming language.

Analogical Model: An analogous model can be used which allows modeling the complex system that is going to be developed. An analogous system is an Enterprise Resource Planning System (ERP), just as in the complex system that is being worked on, there is an objective user that is constantly monitored (patient) to detect any anomaly and in case of any failure (biomedical signals out-side of specific ranges) take the respective control/alert actions to return to the appropriate conditions. All information (patient data) is stored in a database in the cloud for further analysis and possible prediction processes.

In an ERP system, constantly different users with different roles consult the information in the database to use it as input in other processes. As in the complex system, doctors and/or relatives will constantly consult these databases to make decisions.

Conceptual Model: A conceptual model can be used, describing the key elements of the complex system, their characteristics and the different relationships between them:

- $\quad$ Complex system key elements: 
- Patients: people who need to be monitored remotely in their homes for any medical condition.

- The Colombian government (through the ministry of health): in charge of guaranteeing the provision of the health system to $100 \%$ of the Colombian population.

- Patient's relatives: people interested in having information on the status of their loved ones in real-time 7/24.

- Health Promoting Entities (HPE): the link between the government and Colombian citizens in terms of providing health services.

- Institutions providing health services: entities that are in direct contact with patients. They hire doctors from different disciplines to provide services in different specialties.

- SIMORETE (Remote Patient Monitoring System): a project that will be supporting HPE and guarantee quality monitoring to patients who require it.

Graphic Model: the relationships between the main entities that make up the system are graphically identified. In Figure 1 , the main components of the complex system can be observed at a high-level description.

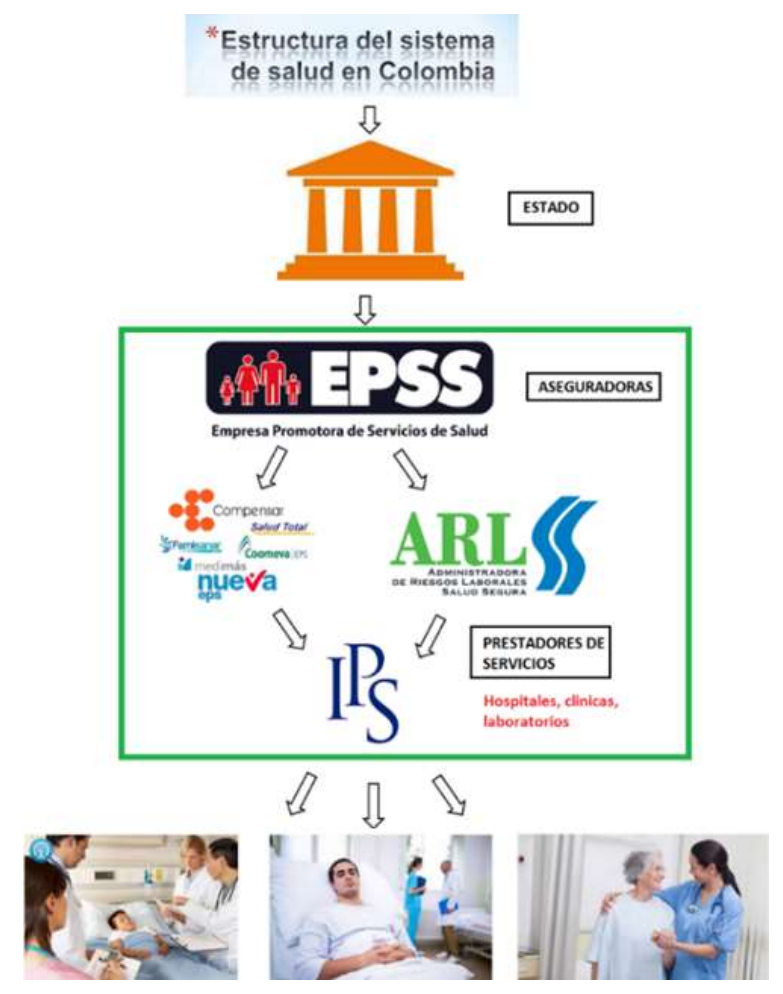

Figure 1: Structure of the Colombian health system

Statistical model: this model can be used to propose an equation or set of equations with different components (technical, financial, social) that allow finding financially viable and sustainable model overtime for the Colombian health model.

Some parameters that should be considered:

- Contribution to health by people

- Contribution to health by companies

- Moderator fee (additional money paid by people each time they go to a general medical appointment, specialist or 
to claim medication)

- $\quad$ Prepaid medicine

- Projections of the state funding budget for the health system

- Technological infrastructure for telemedicine

- The bandwidth is available for people according to their socio-economic situation.

- Several contributors to the health system. Average age / demographic bonus

Process Model: The complex system is analyzed as a process of different actors and relationships. Through the BPMN language, the complex system is modeled using the Enterprise Architect software. This modeling allows us to observe the main and secondary elements of the system and their different relationships. The actors that are part of the model are described below. In Figure 2 you can see the actors and their interactions through the process diagram.

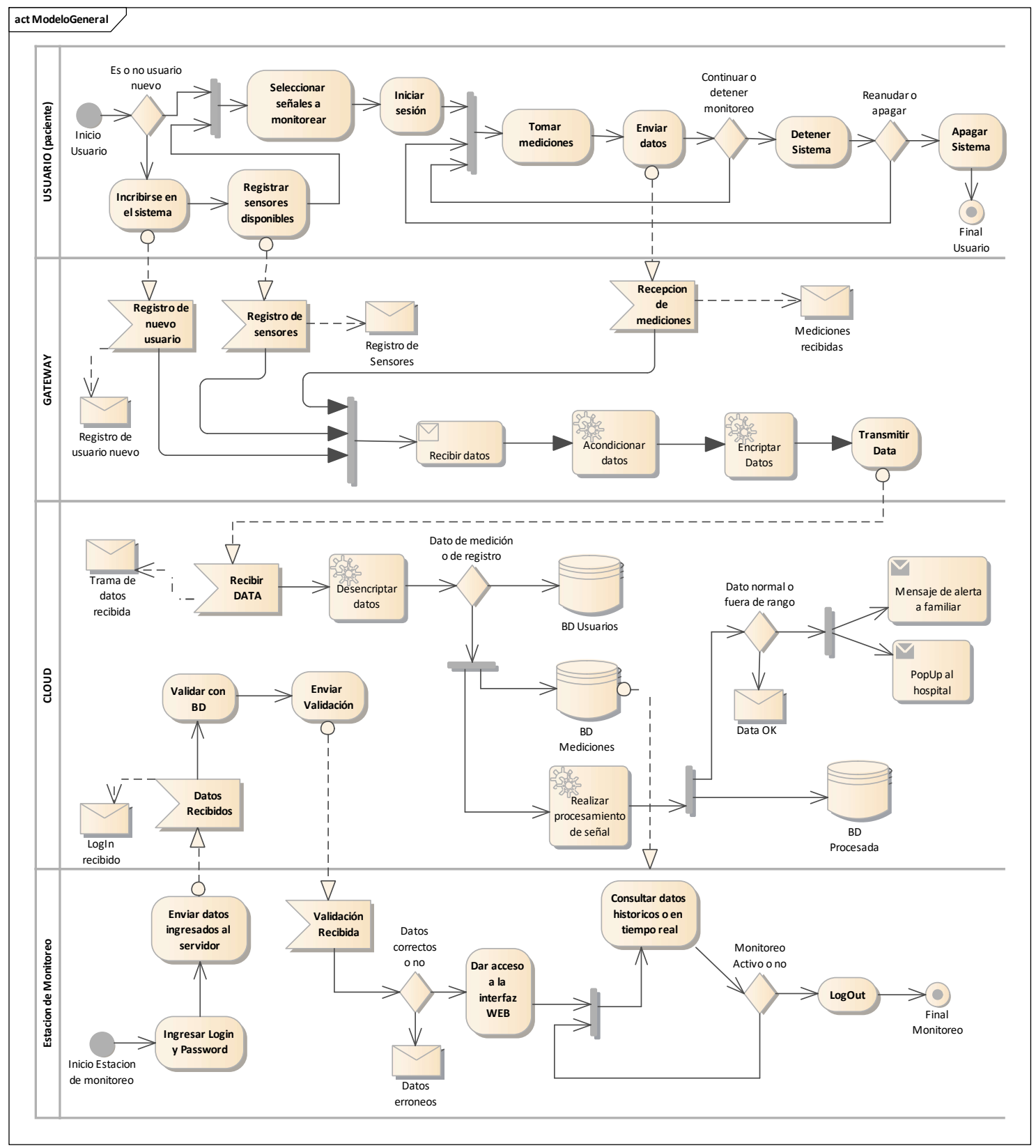

Figure 2: Model of the complex system using processes 
- Actor 1: Patient. They must register in the system and use the sensors that allow them to capture different biomedical signals to send it to the remote monitoring system.

- Actor 2: Remote patient monitoring system (SIMORETE). Responsible for encrypting all information, send it to the server in the cloud, decrypt it, analyze it and take control actions. Additionally, the system must save all this information in a database.

- Actor 3: Monitor stations. They can be doctors enrolled in the system or relatives who are interested in having real-time information of the patient or see historical data to make a more detailed analysis.

\subsection{Development of the Prototype in Python}

The proposed system has three main blocks:

- Block 1: Loading data, in this module the system obtains data from different sources and in different formats, for example: .txt, .csv, .json, among others. At the same time, it obtains real-time information about web services through Python add-ins and libraries.

- Block 2: Conditioning and processing through several Python libraries, among which stand out: wfdb, Numpy, Sympy, Scipy, Pandas and Scikit-learn for machine learning.

- Block 3: Graphs and results, this module makes use of many graphics libraries, among which the Matplotlib library stands out and the graphics complement of the wfdb library.

After having modeled the complex system the design was done by classes in a program in Python. The prototype consists mainly of 6 packages within you can find several associated classes (see Figure 3).

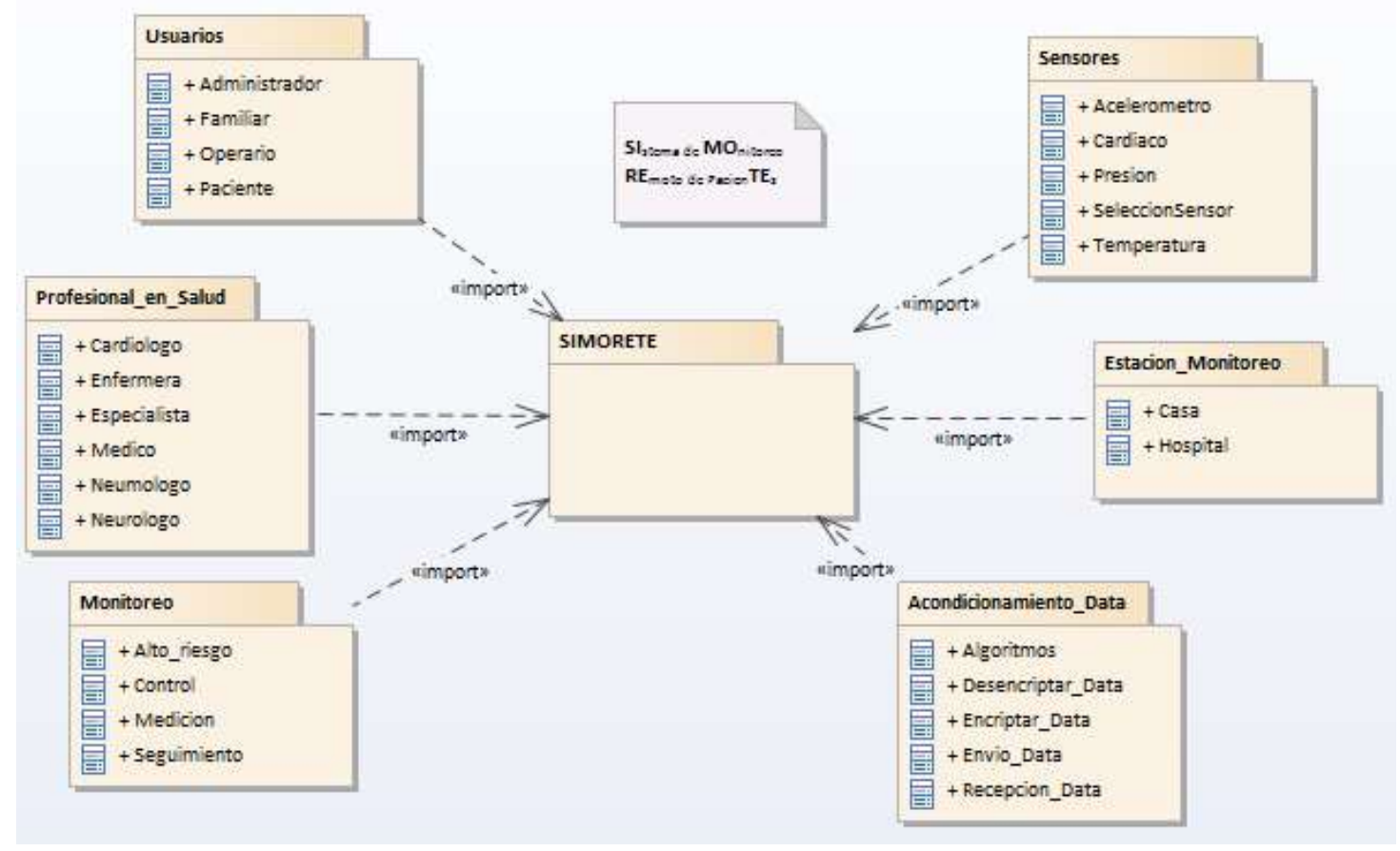

Figure 3: Diagram of packages of the prototype

The main packages are:

- Users 
- Sensors

- Health professionals

- $\quad$ Type of monitoring

- Data conditioning

- Monitoring stations.

After the prototype design in Python, a general outline of the hardware components that the system must have and its interactions with the software was made (Figure 4).

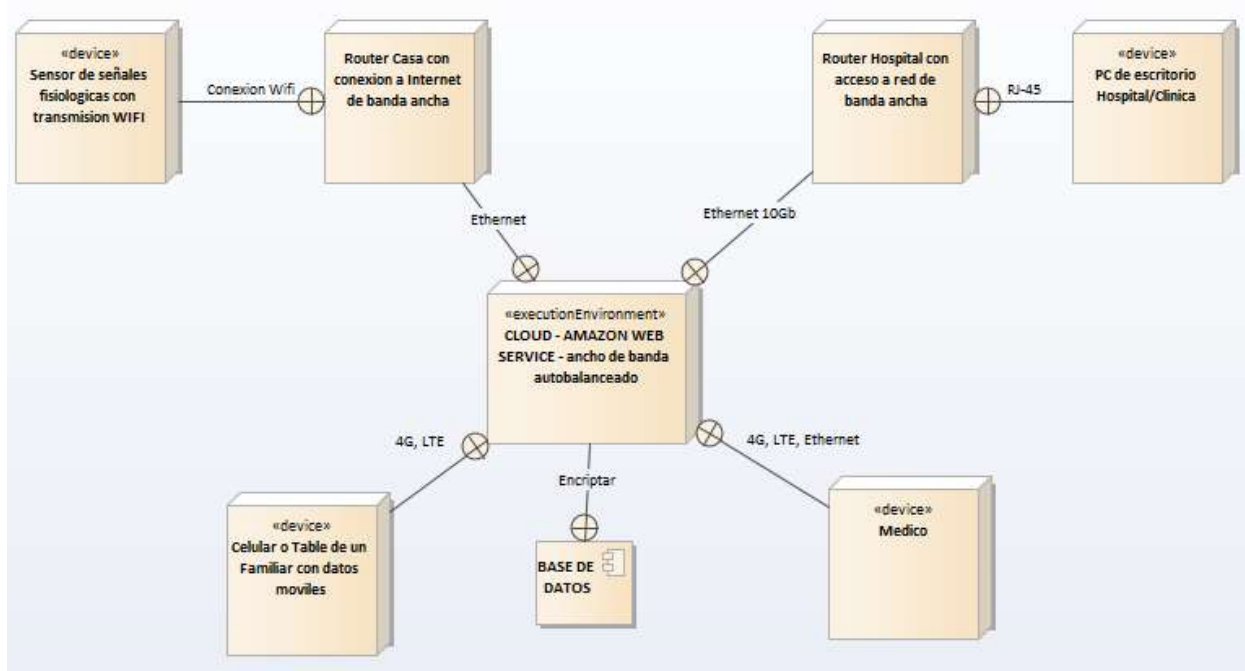

Figure 4: Prototype component diagram

An interaction scheme was also made between the potential users of the system through a diagram of use cases (Figure 5). The main physical elements that the system must have are: different kinds of physiological signal sensors, physical links to the Internet (Router, WiFi), a physical server that was tested through Amazon Web Services and end-user devices such as laptops or smartphones.

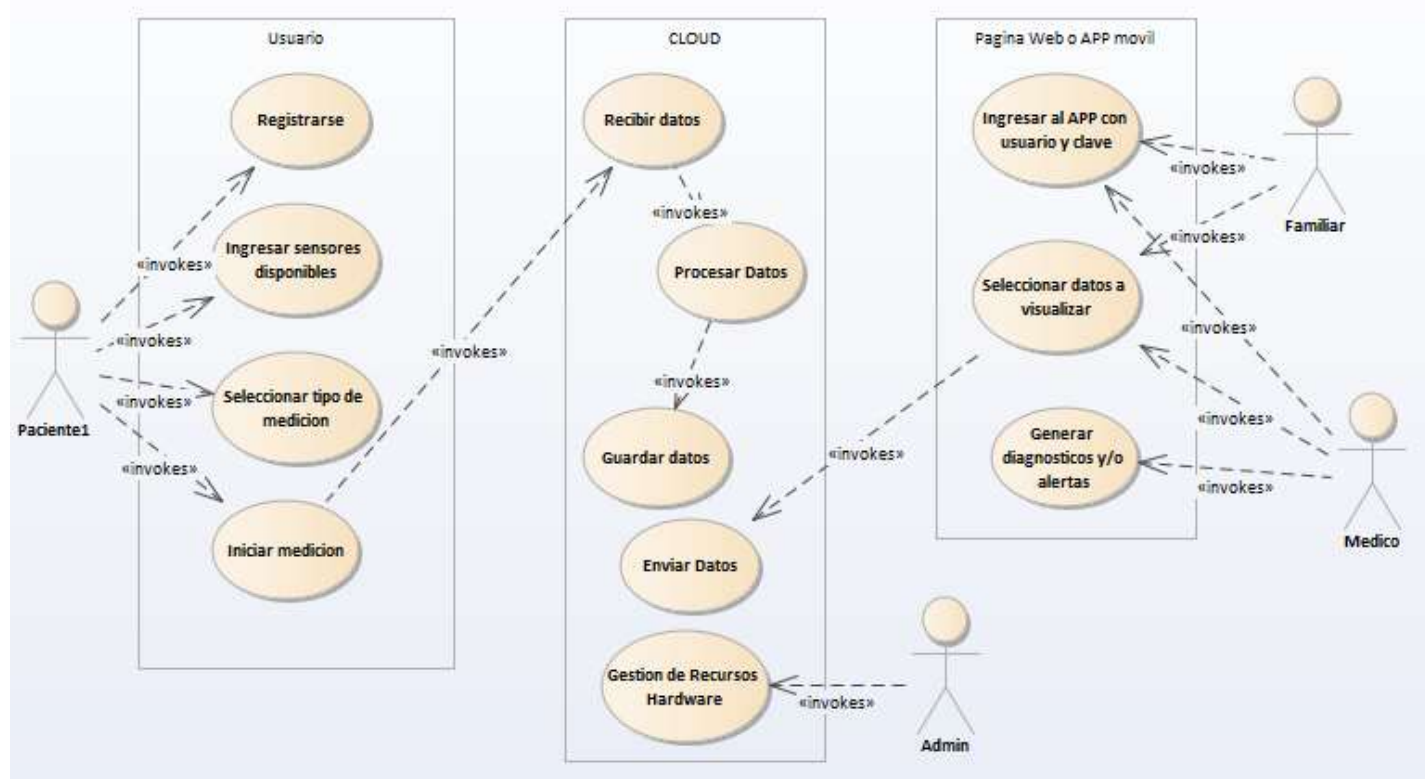

Figure 5: Use case diagram 
The main users of the modeled system are patients, relatives of patients, doctors and health specialists and administrators of the technological platform. The entire system is designed and tested locally. Finally, it is embedded in an Amazon Web Services (AWS) virtual machine and will be available in real-time in the internet cloud.

\section{RESULTS}

Different tests were performed on the prototype using different data sources. These tests were done locally and finally, tests were performed on a server in the cloud. Some of the results obtained are described below:

\subsection{Web Services Data Acquisition Test}

To have current useful information in the platform on topics of telemedicine, good health habits, advice from experts in nutrition and health-related topics, a class was designed to connect to Twitter and filter by keywords related contents and save them in a .json file to be used later. Figure 6 shows the programming baselines and the record of the saved data.

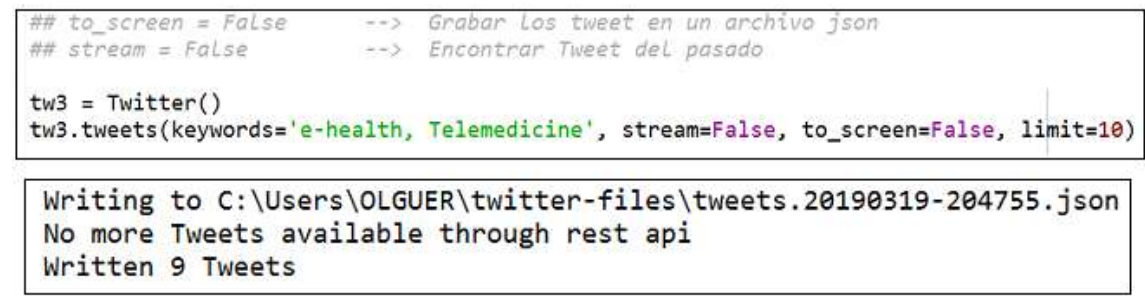

Figure 6: Acquisition of Twitter web service data

\subsection{Biomedical Signal Generation Test}

To compare real signals with a gold standard, a class was designed to create different types of biomedical signals from equations and mathematical models. An example of the signals created can be seen in figure 7 (photoplethysmography signal (PPG)). This signal was generated through a mathematical equation (1), this equation was entered Python using functions of the Sympy library and the graph is performed through functions of the Matplotlib library.

$P P G_{\text {sim }}=[0,05 \cdot \operatorname{sen}(2 \pi f 3 t)+0,4 \cdot \operatorname{sen}(2 \pi f t)+0,25 \cdot \operatorname{sen}(2 \pi f 2 t+45)]+0,5$

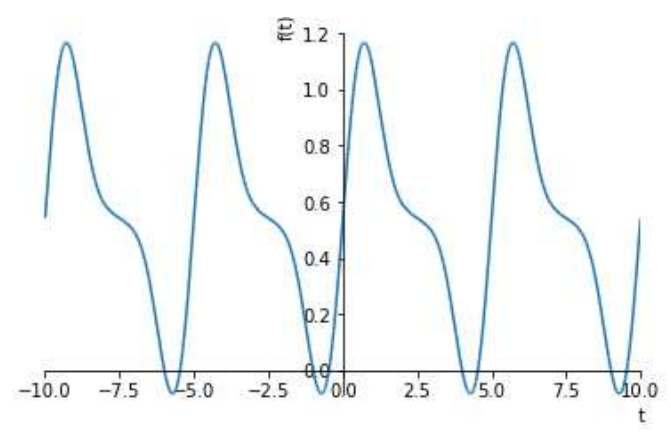

Figure 7: Simulated plethysmography signal

\subsection{Conditioning Test, Processing and Graphics of Biomedical Signals}

All the data acquired from the different databases must be conditioned, processed and graphed. Two working examples of the design classes are mentioned: a class of spectral analysis of electrocardiographic (ECG) signals and an automatic learning class for the classification of hand movements through electromyographic (EMG) signals. 


\subsubsection{Class for Spectral Analysis of Electrocardiographic Signals (ECG)}

The spectral analysis of biomedical signals was performed using functions of the Scipy library, the results were obtained using data from electrocardiographic (ECG) signals.

Data from the Physionet database was used, specifically the file named "100" which contains two channels of ECG signals.

One of the channels has an ECG with Arrhythmia and the other channel has an ECG with normal characteristics. The data was imported and plotted by functions of the waveform-database library (wfdb) (see Figure 8).

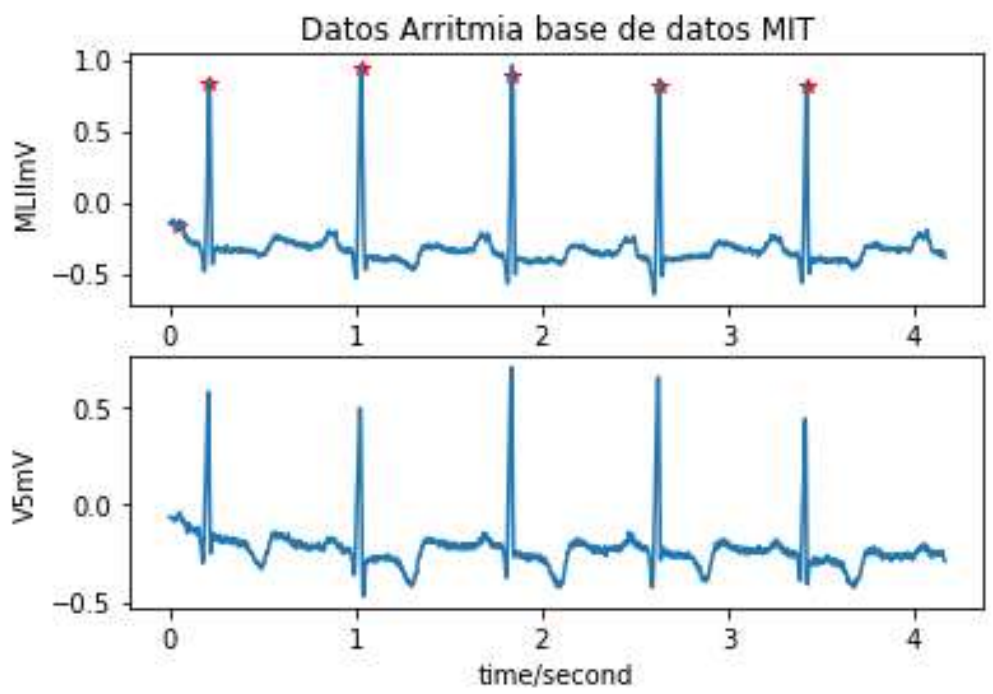

Figure 8: Graph of Electrocardiographic signals

Then enter the appropriate parameters to the FFT (fast discrete Fourier transform) function of the Scipy library is the frequency spectrum of the two EEG signals and a histogram is made with the results obtained to perform analyzes and subsequent observations (see Figure 9).
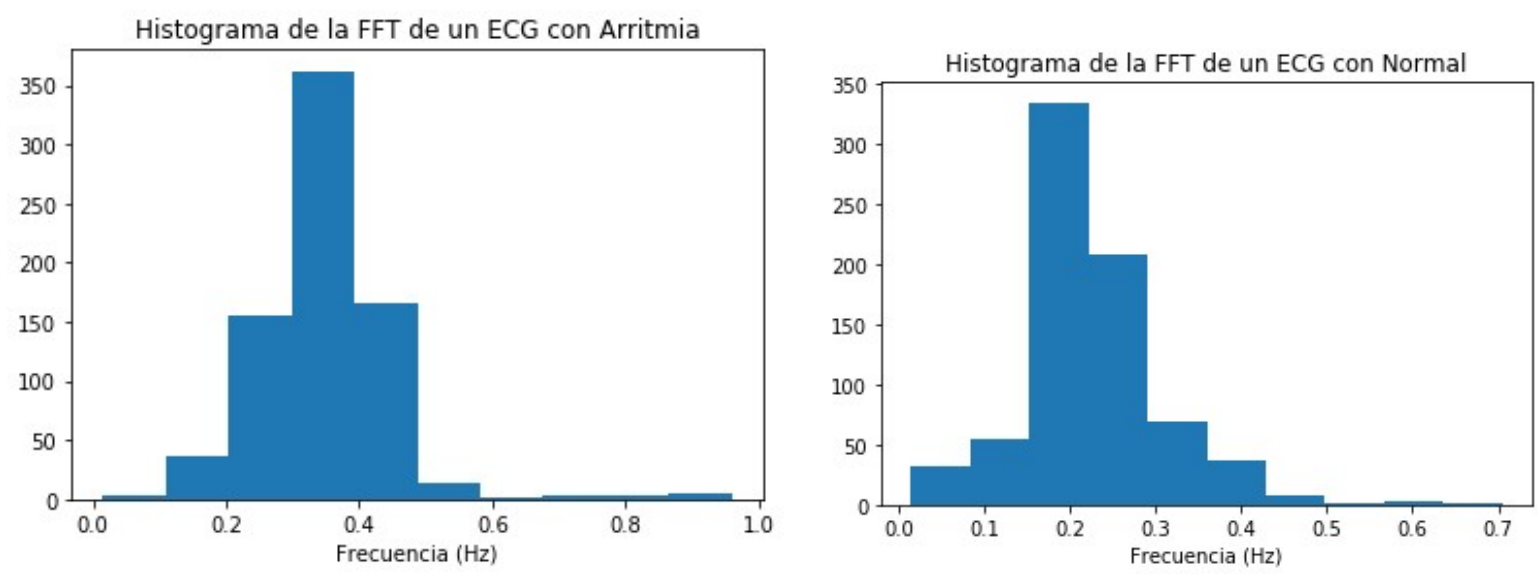

Figure 9: Histogram of the frequency spectrum of electrocardiographic signals 


\subsubsection{Class for machine learning: classification of hand movements using electromyography signals (EMG)}

A Dataset called "EMG Data for Gestures Data Set" was used, which contains a record of electromyography signals from 36 volunteers. Each file of a volunteer is made up of 10 columns with the description shown in Table 1.

\section{Table 1: EMG Data for Gestures Data Set}

\begin{tabular}{|l|l|}
\hline Column & Description \\
\hline 1 & Time in $\mathrm{ms}$ \\
\hline 2 to 9 & EMG Channels \\
\hline 10 & Data Label \\
\hline
\end{tabular}

Table 2 shows the detail of the information contained in the Data Label space.

Table 2: Detail of the Data Label

\begin{tabular}{|l|l|}
\hline \multicolumn{2}{|c|}{ DATA LABEL } \\
\hline LABEL & DESCRIPTION \\
\hline 0 & Unchecked data \\
\hline 1 & Resting hand \\
\hline 2 & Hand clenched in a fist \\
\hline 3 & Bending of the wrist \\
\hline 4 & Wrist extension \\
\hline 5 & Radial deviations \\
\hline 6 & Ulnar deviations \\
\hline 7 & Extended palm \\
\hline
\end{tabular}

The data is imported through the class and saved as a Panel to make use of the potential of the Pandas library. The basic statistics of the Data Set are calculated, and the results shown in Figure 10 were obtained.

$\begin{array}{|lrrcrr|} & \text { channel1 } & \text { channel2 } & \ldots & \text { channel8 } & \text { class } \\ \text { count } & 343038.000000 & 343038.000000 & \ldots & 343038.000000 & 343038.000000 \\ \text { mean } & -0.364127 & -0.425427 & \ldots & -0.575789 & 3.550548 \\ \text { std } & 17.944188 & 13.961548 & \ldots & 18.297931 & 1.746432 \\ \text { min } & -128.000000 & -128.000000 & \ldots & -128.000000 & 1.000000 \\ 25 \% & -0.000050 & -0.000050 & \ldots & -0.000060 & 2.000000 \\ 50 \% & -0.000010 & -0.000010 & \ldots & -0.000010 & 4.000000 \\ 75 \% & 0.000040 & 0.000030 & \ldots & 0.000040 & 5.000000 \\ \text { max } & 127.000000 & 127.000000 & \ldots & 127.000000 & 7.000000\end{array}$

Figure 10: Statistics of the Data Set "EMG data for gestures"

When making the graphs of the different channels in a two-dimensional plane, it can be observed that the data are very overlapping, which makes separation and identification quite difficult. Due to this characteristic of the data, it is decided to make a principal component analysis (PCA) to detect the main patterns of the Data Set using the functions of the Scikit-learn library (see Figure 11). 


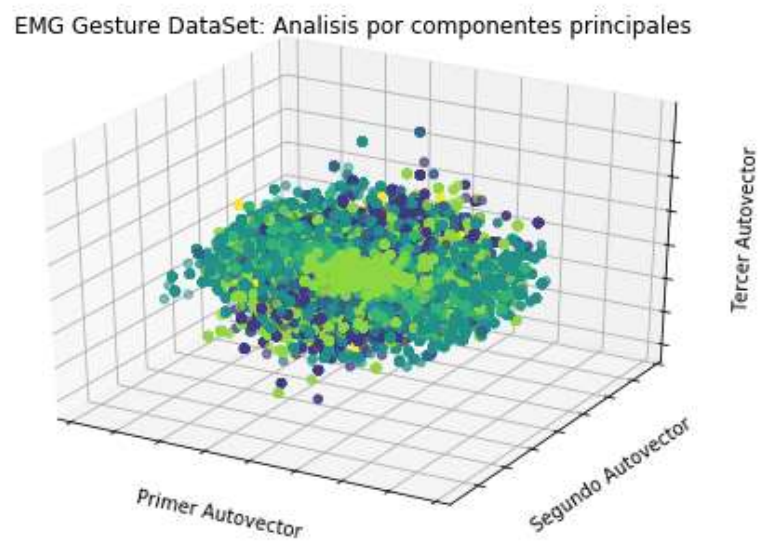

Figure 11: Result of the PCA made to the Data Set

\subsection{Tests in the Internet Cloud}

A professional account was created in Amazon Web Services (AWS) and a server machine was created where the free distribution of Linux was installed. Subsequently, Anaconda was installed on the server and the correct performance of the classes that make up the prototype was verified.

The classes were tested on the AWS server in real-time. The instructions are sent to the server through an open console through an SSH connection. In this class, a large volume of data is imported, processed through the functions of the Pandas library and reports of the results obtained are generated.

\section{DISCUSSIONS}

In this section, a comparison of the literature with the prototype proposed in this document is made. The positive aspects and the most relevant contributions are highlighted, and the advantages of the proposed model compared to these solutions are stated.

Joshi, Saxena, Godbole, \& Shreyad [3] identify six pillars to develop a frame of reference: Social, Administration, Economic, Legal, Technology and Sustainability (SMELTS), the article describes how the integration of these factors could make the initiative of smart cities be a successful project, however, they do not describe how these elements interact when making a technological implementation.

Barth et al. [4] present a conceptual framework conformed by seven pillars: in-formation and knowledge, infrastructures, economy, politics (electronic governance) and administration (electronic government), spaces (spaces of services and spaces of places), location factors, the behavior of information of people, and problem areas. In this case hardware or software, technological components are not detailed, and there is no practical validation of the proposed framework.

The work developed by Vlasios [5], which has the support of the United Nations, it proposes a frame of reference where they involve technological aspects and detail parameters of communication protocols. At the same time, they make a state of the art of the main tools used for the implementation of the internet of things. However, they do not contemplate factors of real-time processing design, nor the impact of an adequate use of bandwidth. 
In [6] they describe and determine the relevance of the adequate handling of large volumes of information (Big Data) by applying it to two cases of study: analysis of medical images and bioinformatics. As an important contribution they use and mention tools for handling large volumes of data in conjunction with data mining tools. They mainly work on the processing layer but do not address issues of acquisition and presentation of results in local interfaces or web interfaces in the cloud.

In [7] they do the performance evaluation and dimensioning of a convergent access network, based on MetroEthernet standards, with a high number of devices (terminals, smartphones and IoT devices) that inject traffic into the environment of a Smart City. This analysis is carried out in a simulation environment through the SimulCity program. As the main contribution, they propose with a methodological approach, some key indicators of medical services associated with citizen welfare and delay analysis for services in real-time. This work contemplates the first important step in the modeling of complex systems of telemedicine platforms: the transmission of high volumes of data, however, some external parameters must be modeled in the following phases.

In [8] and [9] they propose a real-time monitoring system through an Arduino module, a temperature and heart rate sensor. In [8] they use a GSM wireless trans-mission module and a GPS positioning module. The information from the sensors is sent to a platform developed using HTML5, CSS, PHP and a database developed in MySQL. As the main contribution, they use wireless transmission technologies and geolocation. In [9] they perform data storage locally using an SDCard and develop a mobile application for Android to visualize the information.

Neither of the two proposed systems performs information processing either locally or in the cloud. They do not perform a data traffic analysis which presents a relevant challenge when are analyzed systems that are going to be used by many people at the same time continuously.

In [10] they propose an architecture for a monitoring system in smart cities based on the Internet of things. The main contribution is the identification of the main network requirements in a monitoring system in terms of updating realtime events, bandwidth requirements and data generation. This architecture provides key guidelines for the implementation of telemonitoring platforms which are also considered in the present work.

In [11] they propose an architecture based on the $\mathrm{NGeH}$ (next-generation e-Health) paradigm as an extension of the ETSI (European Telecommunications Standards Institute) standard. The proposed architecture integrates mechanisms of sensor networks, profiling mechanisms and security mechanisms that allow to easily take advantage of the central capacities so that reliable NGeH services can be built. The main contribution is made through the implementation of an eHealth telemonitoring service in an intelligent home environment applying the proposed architecture. This architecture contemplates several essential elements for the implementation of telemedicine platforms including an international standard.

\section{CONCLUSIONS}

There are many more initiatives, proposals and approaches to developments of biomedical systems that implement new technologies, but all are general, and no work has been found aimed at telemonitoring services or dynamic processing of biomedical signals. In the present work, a holistic prototype was designed to consider the internal and external factors of the hardware and software that are involved in the telemonitoring process. The prototype is equipped with tools to perform several agile validation tests both in local environments and in the cloud in a fast and scalable way. 
The system modeled through BPMN and UML diagrams manages to generate a complete prototype, which evaluates the main components of signal acquisition, processing and generation of valid results to allow a group of experts to generate actions according to the profile and information obtained from the data of each patient.

The prototype developed in the present work allows us to evaluate the essential technical and structural factors of a remote monitoring platform in real-time.

\section{ACKNOWLEDGMENTS}

Special thanks to the Universidad Distrital Francisco José de Caldas for their support in the development of this project.

\section{REFERENCES}

1. El Espectador. (2018). Colombia se está envejeciendo: el 9,23\% de la población tiene más de 60 años. Retrieved from: https://www.elespectador.com/noticias/economia/colombia-se-esta-envejeciendo-el-923-de-la-poblacion-tiene-mas-de-60anos/

2. Berrio Valencia, M. I. (2012). Envejecimiento de la población: un reto para la salud pública. Colombian Journal of Anesthesiology. 40(3):192-194.

3. Joshi, S., Saxena, S., Godbole, T. \& Shreya. (2016). Developing Smart Cities: An Integrated Framework. Procedia Computer Science. 93:902-909.

4. Barth, J., Fietkiewicz, K., Gremm, J., Hartmann, S., Ilhan, A., Mainka, A., Meschede, C. \& Stock, W. (2017). Informational Urbanism. A Conceptual Framework of Smart Cities. In Proceedings of the 50th Hawaii International Conference on System Sciences, p.p. 2814-2823, 2017.

5. Vlasios, T., Pramod. A., Payam, B., Marten, F., Frieder, G., Muhammadm, I., Sefki, K., Daniel, K., Alessandra, M., Dan, P., Ralf, T. \& Thorben, I. (2013). Real-Time IoT Stream Processing and Large-scale Data Analytics for Smart City Applications. City Pulse.

6. Kumaran Nair, S. S. \& Ganesh, N. (2016). An exploratory study on Big data processing: a case study from a biomedical informatics. 3rd MEC International Conference on Big Data and Smart City, p.p. 1- 4, 2016.

7. Gómez-Sacristan, A. \& Hernández-Rodríguez, M. A. (2018). Communications for Sanitary Teleservices in a Smart City. 2018 Global medical engineering physics exchanges, p.p 1- 4, 2018.

8. Aziz, K., Tarapiah, S., Ismail, S. H. \& Atalla, S. (2016). Smart Real-Time Healthcare Monitoring and Tracking System using GSM/GPS Technologies. 3rd MEC International Conference on Big Data and Smart City, p. 7, 2016.

9. Khan, T. \& Chattopadhyay, M. K. (2016). Smart Health Monitoring System. IEEE, International Conference on Information, Communication, Instrumentation and Control (ICICIC 2017), p. 6, 2017.

10. Khoi, N. M., Saguna, S., Mitra, K. \& Ahlund, C. (2015). IReHMo: An Efficient IoT-Based Remote Health Monitoring System for Smart Regions. 17th International Conference on E-health Networking, Application \& Services (HealthCom), p. 6, 2015.

11. Fengou, M.-A., Mantas, G., Lymberopoulos, D., Komninos, N., Fengos, S. \& Lazarou, N. (2013). A New Framework Architecture for Next Generation e-Health Services. IEEE Journal of biomedical and health informatics. 17(1):9-18. 